EPJ Web of Conferences 45, 01110 (2013)

DOI: $10.1051 /$ epjconf $/ 20134501110$

(C) Owned by the authors, published by EDP Sciences, 2013

\title{
Stereoscopic reconstruction of 3D PIV data in T-junction with circular profile
}

\author{
M. Kotek ${ }^{1}$, D. Pavlík ${ }^{2}$, V. Kopecký², and D. Jašíková ${ }^{1}$ \\ ${ }^{1}$ Institute for Nanomaterials, Advanced Technologies and Innovation, Technical University of Liberec, 46117 Liberec, \\ Czech Republic, michal.kotek@tul.cz \\ ${ }^{2}$ Faculty of Mechatronics, Informatics and Interdisciplinary Studies, Technical University of Liberec, 46117 Liberec, \\ Czech
}

\begin{abstract}
In this paper experimental study of flow in T-junction using 3D PIV method is presented. Motion of seeding particles was recorded by a pair of suitably located cameras in precisely defined cross sections of the junction. Based on this information, three-dimensional model of flow in different sections of junction was reconstructed. The reconstruction results from the projection matrixes of each camera, which are obtained from positions of objects in the scene and their projection positions in the image plane. Standard 3D PIV reconstruction was rejected, because of optical distortion in T-Junction.
\end{abstract}

\section{Introduction}

T-junction is illustrated on figure 1. This usual element in pipeline nets leads to unsteady structures of flow and energy losses. These are the subjects for this experimental research on water. The measurement results are used to verify the mathematical model which describes the flow inside junction, refer to [1].

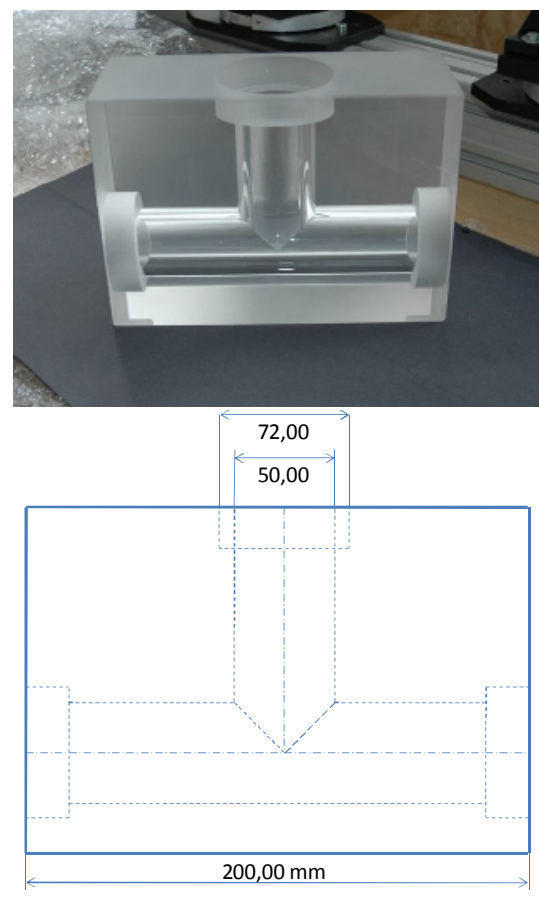

Fig. 1. Glass model of circular T-junction
Research is performed using 3D PIV system in precisely defined cross sections of the junction. Than is reconstructed three-dimensional flow model for each section. The algorithm which is reconstructed on the basis of the projection matrices of each camera which are obtained from positions of objects in the scene and their positions in the image plane is created. Commercial programs such as the Dynamic Studio perform this reconstruction but their algorithms are not sufficiently robust. The algorithm failure in the presence of significant image distortion that is caused by observing objects in cylindrical glass.

\section{Principle of 3D PIV}

The method is based on the stereoscopic reconstruction, very similar to human sight principle. Two digital cameras are used for recording, each camera monitors from different angle the region of interest illuminated with laser cut, see figure 2. Cameras record different images of two-dimensional flow field. Based on this information three-dimensional movement of seeding particles is reconstructed. Compared to conventional (2D) method it is possible to determine the component shift (velocity) perpendicular to the measuring plane defined by the laser cut. However, if the cut is really "thin" plane, it is not possible to record the aforementioned component of shift. The cut must have thickness at least $5 \mathrm{~mm}$, where laser light intensity does not decrease $50 \%$ of the maximum value [3]. Parameters of cut, as well as its 
thickness, are defined by the cylindrical optics and beam expanders (beam expanding optics).

Axis of camera lens clutched angle is seen on figure 2 . This setup achieved the most accurate determination of shift vector. With the reduction of the angle the accuracy of the method decreases.

The camera's lenses used for these measurements have a very thin depth of sharpness. To keep the recorded objects focused in the whole image plane (CCD detector), Scheimpflug condition must be executed.

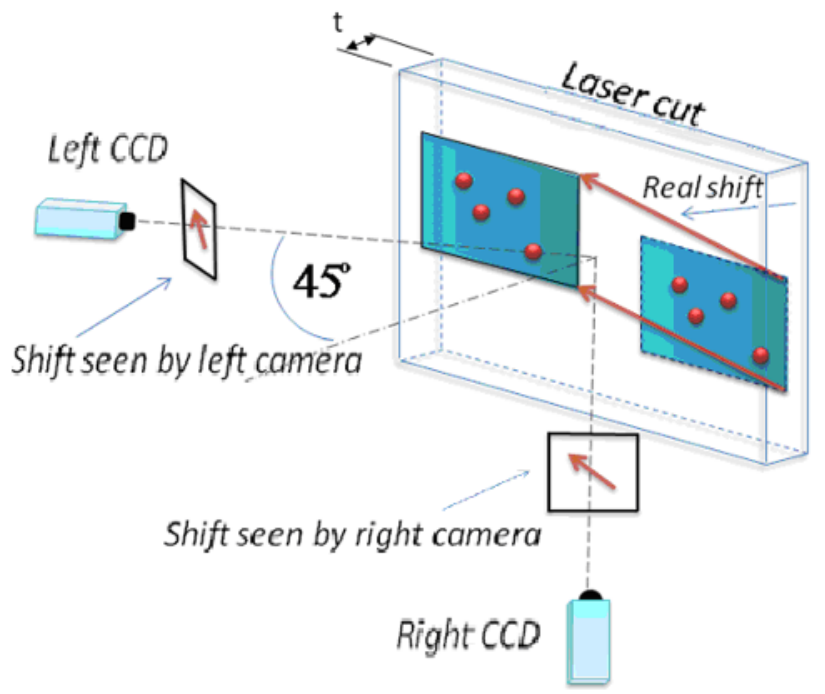

Fig. 2. Principle of $3 D$ PIV

Recording and image processing of particles is the same as for 2D measurements. Images captured by the left and right camera are processed separately, so primarily are obtained velocity vector maps for the left and right camera. If the projection matrices of cameras are known, it is possible to reconstruct the final 3D velocity vector map, corresponding to the real shift of particles in the measuring plane [3].

\section{Experimental setup}

The T-junction is connected to an open branching pipeline system with a pump and tank, see "figure 3". The water flowing to each branch of junction is regulated by valves, behind them are installed flow meters and two meters long pipe to stabilize the flow before entering the $\mathrm{T}$-junction. The water flow is distributed to the branches of T-junction in several modes. On the figure 4 the flow directions and the marking of these modes are illustrated. In each of the four modes, the total flow in junction is set to $5.51 / \mathrm{s}$.

Polyamide particles with a diameter covered with rhodamine (Nile Red from Spherotec Inc.) are used for flow seeding. Rhodamine is a fluorescent substance which during excitation of laser beam (532 nm green) emits radiation corresponding to the orange $(570 \mathrm{~nm})$. The camera lenses are fitted with a filter, that transmits only the orange light. Laser light reflections from walls and bubbles inside the flow are eliminated.

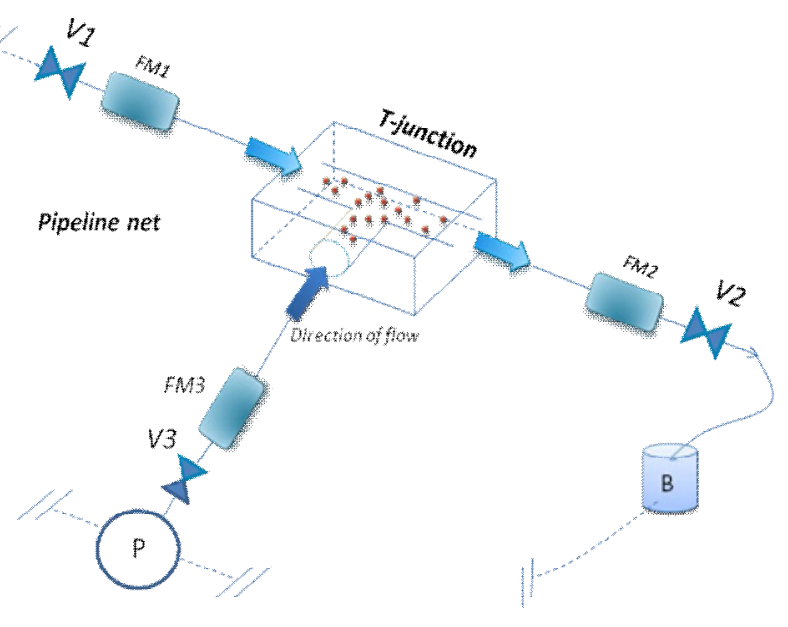

Fig. 3. Pipeline system (V-valves, FM-flowmeters, P-pump, B-basin)

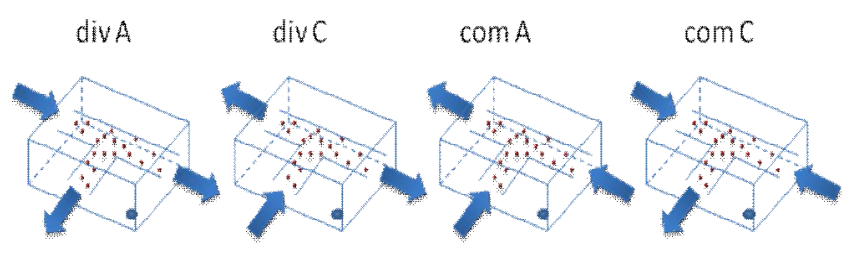

Fig. 4. Flow modes

On the figure 5 camera and laser placement is illustrated. Laser cut defines the measurement plane, CCD cameras record the measuring plane from opposite site. Dantec Hisense CCD cameras with a resolution 1280 by 1024 pixels and 12 bit brightness resolution were used for image recording. As lighting equipment NewWave Gemini pulse laser is used.
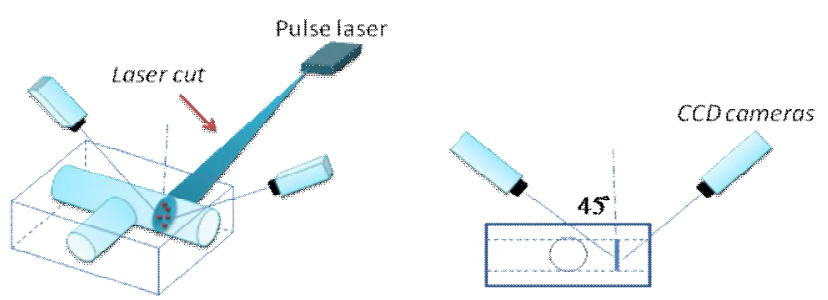

Fig. 5. Position of laser and cameras

\section{Calibration}

Precise calibration procedure precedes the measurement. This procedure helps to find suitable image model describing the transformation between points in space (points of calibration pattern) and their projections in the image planes (photos). The calibration target on figure 6 is attached on a specially manufactured breech. Breech allows a shift of target along the pipeline in the $\mathrm{T}$ junction model filled with water, to keep the real refraction indexes and optical distortion. 
Cameras record the calibration target in several positions. Records of calibration patterns are used to find the projection matrices of each camera. The same procedure is performed for the calibration target placed in all model branches.

Synchronization of a pulsed laser and camera is controlled by the programming environment Dynamic studio.

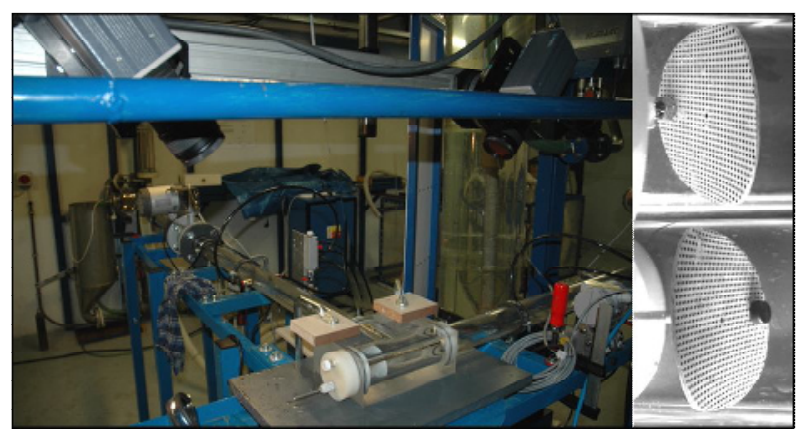

Fig. 6. Calibration setup and targets

\section{Calibration}

For 3D reconstruction the perspective model of camera is considered. This model transforms coordinates of the object X (dots of calibration target) into coordinates of the projective space $\mathrm{x}$ (dots on the image) according [3]:

$$
\boldsymbol{x}=P \boldsymbol{X},
$$

where $P$ is the projective matrix with the size $3 \times 4$ and the matrix rank 3 . Because the mathematical parameters of the camera and its lens were not defined, so the projective matrix was determined from the coordinates of dots on target and on the image.

Projective matrix is defined as below:

$$
\left[\begin{array}{c}
k u \\
k v \\
k
\end{array}\right]=\left[\begin{array}{llll}
P_{11} & P_{12} & P_{13} & P_{14} \\
P_{21} & P_{22} & P_{23} & P_{24} \\
P_{31} & P_{32} & P_{33} & P_{34}
\end{array}\right] \cdot\left[\begin{array}{c}
X \\
Y \\
Z \\
1
\end{array}\right]
$$

If the position of at least six point in object plane $X$, the coefficient to could be find, this procedure was described in [3].

Projective matrix $\mathrm{P}$ is expressed: $A P=0$

where $\mathrm{A}$ is:

$$
A=\left(\begin{array}{cccccccccccc}
X_{1} & Y_{1} & Z_{1} & 1 & 0 & 0 & 0 & 0 & -u_{1} X_{1} & -u_{1} Y_{1} & -u_{1} Z_{1} & -u_{1} \\
0 & 0 & 0 & 0 & X_{1} & Y_{1} & Z_{1} & 1 & -v_{1} X_{1} & -v_{1} Y_{1} & -v_{1} Z_{1} & -v_{1} \\
\vdots & \vdots & \vdots & \vdots & \vdots & \vdots & \vdots & \vdots & \vdots & \vdots & \vdots & \vdots \\
X_{n} & Y_{n} & Z_{n} & 1 & 0 & 0 & 0 & 0 & -u_{n} X_{n} & -u_{n} Y_{n} & -u_{n} Z_{n} & -u_{n} \\
0 & 0 & 0 & 0 & X_{n} & Y_{n} & Z_{n} & 1 & -v_{n} X_{n} & -v_{n} Y_{n} & -v_{n} Z_{n} & -v_{n}
\end{array}\right)
$$

and are coordinates of point $\mathrm{n}$ in the world coordinate system, are coordinates of point $\mathrm{n}$ in the image plane. Projective matrix $\mathrm{P}$ was found with the Singular value decomposition (SVD) of the matrix A.
To calculate the projective matrix the coordinates of several points in the scene and on the image must be defined. The calibration targets and its images captured with digital cameras are used for this purpose. Coordinates of the points in the scene are described in the world coordinates system. The orientation of axes of world and image coordinates systems are sketched on the figure 7. Also the dimensions of the calibration target and it movement is described there. The calculation of projective matrix is performed for both cameras.

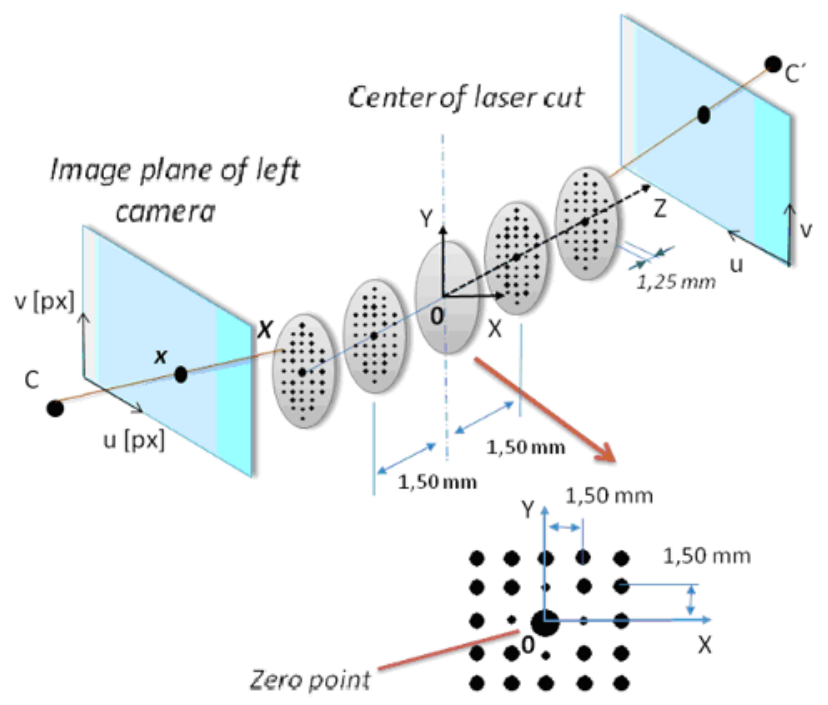

Fig. 7. Coordinate system

Using all this information and the triangulation [4] the three component vector map of velocities is calculated.

\section{Reconstructed results}

The flow field in the area of interest (lighted by a laser sheet) is characterized with a vector map on figure 8 . The results are represented with the velocity scalar map (velocity length).
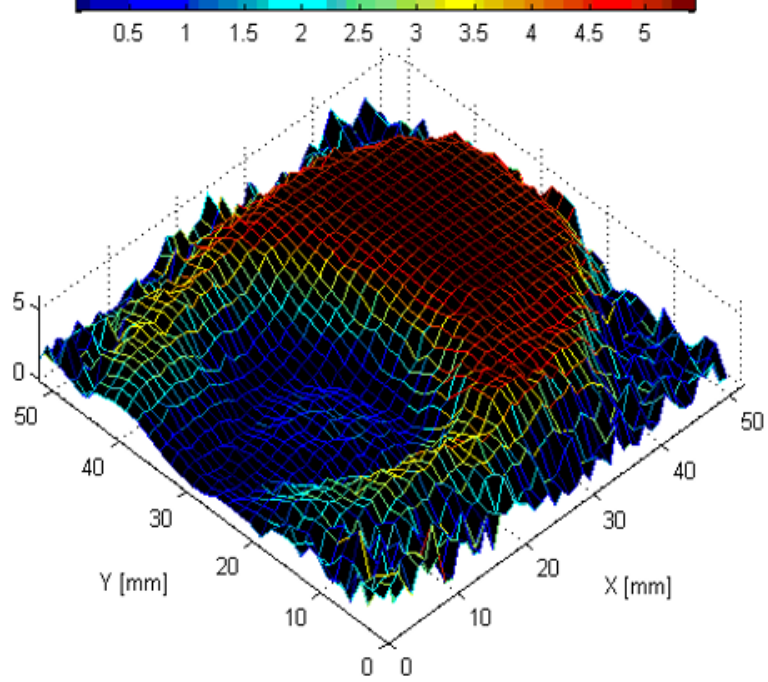

Fig. 8. Flow velocity in the cuts A, ComA (B60\%, C40\%) 
Flow disturbances, the float away behind the T-junction and the flow settling are determined from the $3 \mathrm{D}$ vector maps. The examples of resulted vector maps are represented on figure 9 .

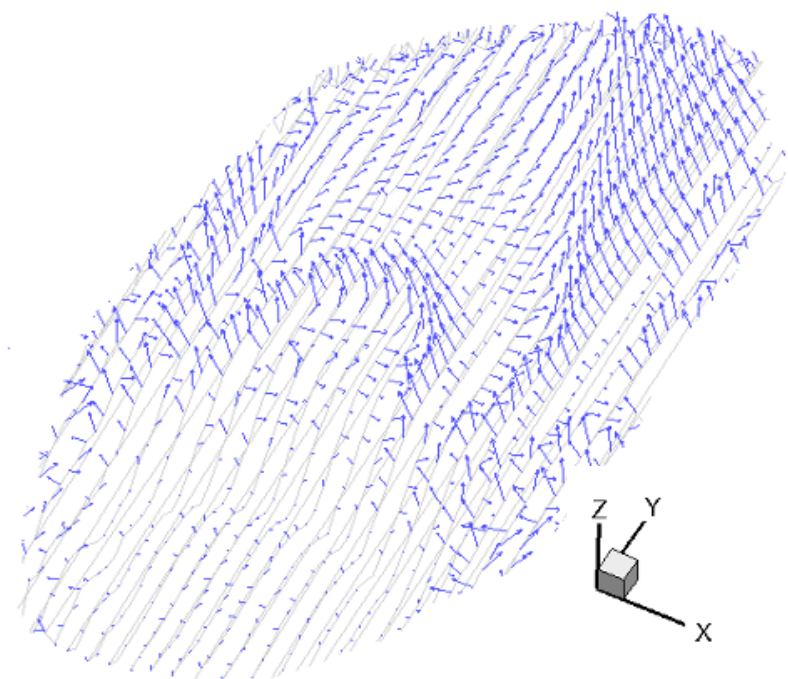

Fig. 9. Three-dimensional map of velocity vector demonstrating the flow in regime $\mathrm{ComA}(\mathrm{B} 60 \%, \mathrm{C} 40 \%)$

These results of 3D PIV measurement of separated branch complete the previous experimental research of the 2D IV measurement in the whole T-junction. Streamlines and velocity value is presented on figure 10 in the same regime and mode as the previous 3D PIV result described on figure 9.

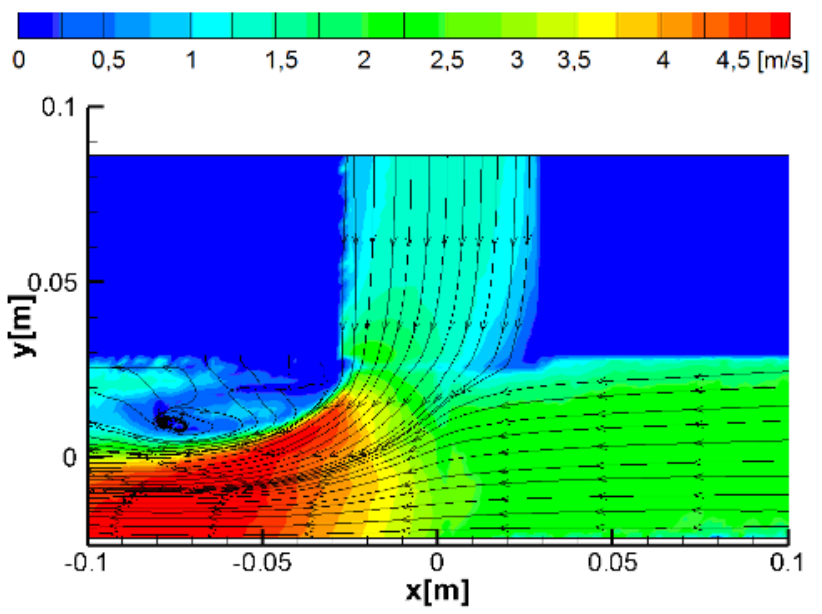

Fig. 10. Flow in T-junction in the regime ComA (B60\%, C40\%)

\section{Conclusions}

For the data analyzing and 3D reconstruction new method was performed. Because of optical distortion in the image of T-junction the standard evaluating methods for 3D PIV did not work properly. Method is based on image preprocessing, determining the mathematical model of camera and lens system and 3D vector reconstruction. This method will be used for the complete analyzing of PIV data of all regimes and modes of the flow in T- junction. With the help of these results the CFD and mathematical simulations will be validated and optimised.

\section{Acknowledgements}

The research reported in this paper was supported in part by the Project OP VaVpI Centre for Nanomaterials, Advanced Technologies and Innovation CZ.1.05/2.1.00/01.0005 and by the Project Development of Research Teams of R\&D Projects at the Technical university of Liberec CZ.1.07/2.3.00/30.0024, by the GACR - project num. GA101/09/1539 and Students research support

\section{References}

1. J. Štigler, Tee junction as a pipeline net element. A new mathematical model Journal of Mechanical Engineering 57 (5), 249-270 (2006)

2. Dantec Dynamics. 3D stereoscopic PIV Reference manual. Denmark : Dantec Dynamic A/S, 2006. 22 p. $99040 \mathrm{U} 4115$.

3. D. Bardsley, B. Li, 3D Reconstruction Using the Direct Linear Transform with a Gabor Wavelet Based Correspondence Measure. Technical Report [online]. 2004, [cit. 2011-05-03]. Available from: $<$ http://bardsley.org.uk/wp-content/uploads/2007/02/3dreconstruction-using-the-direct-linear-transform.pdf $>$. 4. B. Turoňová, Rekonstrukce 3D scény ze stereo obrázků, bachelor's thesis, Charles University of Prague, 2008) 